\title{
LA ENSEÑANZA Y EL APRENDIZAJE EN EDUCACIÓN FÍSICA, UN ESTADO DEL ARTE DE LA PRODUCCIÓN CIENTÍFICA EN IBEROAMÉRICA ENTRE 2005 Y 2016
}

\author{
TEACHING AND LEARNING IN PHYSICAL EDUCATION, A STATE OF THE ART OF SCIENTIFIC \\ PRODUCTION IN IBERO-AMERICA BETWEEN 2005 AND 2016
}

\author{
O ENSINO E A APRENDIZAGEM NA EDUCAÇÃO FÍSICA, UM ESTADO DA ARTE DA PRODUÇÃO \\ CIENTÍFICA EM IBERO AMÉRICA ENTRE 2005 E 2016
}

John Fredy Ariza Jiménez ${ }^{1}$
John Jairo García Díaz

\section{Resumen}

\begin{abstract}
Este artículo es resultado de una investigación que se planteó como objetivo elaborar un estado del arte sobre la producción de conocimiento científico en torno a la enseñanza/aprendizaje de la educación física en el contexto iberoamericano, entre los años 2005 y 2016. La primera parte presenta la metodología. Se realizó una búsqueda de la producción científica en diferentes bases de datos mediante frases compuestas por palabras clave; se aplicaron criterios de inclusión restringiendo la búsqueda a los idiomas español, inglés y portugués y al periodo comprendido entre los años 2005 y 2016. Los artículos científicos seleccionados se sometieron a una matriz de análisis categorial para el correspondiente proceso interpretativo. En la segunda parte, se presentan los resultados. En cuanto a las concepciones de la educación física, se evidenció principalmente una inclinación favorable hacia las tendencias sociomotricidad y actividad física y salud y algunas detracciones frente a la tendencia físico-deportiva. En lo referente a cómo se producen los procesos de enseñanza/aprendizaje, se destaca la evolución hacia los modelos constructivistas. Los hallazgos permiten evidenciar el fortalecimiento de algunas concepciones de la educación física (incluyendo la psico-motricidad y habilidades motrices básicas y la expresión corporal) que toman más fuerza en el contexto Iberoamericano, al igual que la apertura de la asignatura hacia modelos pedagógicos que brindan mayor protagonismo a los estudiantes en el proceso de enseñanza/aprendizaje.
\end{abstract}

Palabras clave: educación física; didáctica; aprendizaje; enseñanza; tendencias de la educación física; corrientes pedagógicas; modelos pedagógicos

\footnotetext{
1 Magister en Educación, especialista en Gerencia y Proyección Social de la Educación, licenciado en Educación Física. Docente investigador Corporación Universitaria CENDA. Correo electrónico: john.ariza@cenda.edu.co

2 Magister en Educación, licenciado en Educación Física. Docente investigador Corporación Universitaria CENDA. Correo electrónico: john. garcia@cenda.edu.co
} 


\section{Abstract}

This article is the result of a research aimed at elaborating a state of the art of the production of scientific knowledge about the teaching/learning of physical education in the Ibero-American context between the years 2005 and 2016. The first part presents the methodology used. A search of the scientific production in different databases was made through phrases consisting of keywords; inclusion criteria were applied, restricting the search to the Spanish, English and Portuguese languages and to the period between 2005 and 2016. The scientific articles selected were submitted to a categorical analysis matrix for the corresponding interpretative process. The second part presents the results of the research. Regarding the conceptions of physical education, we discovered a favorable inclination towards sociomotricity and physical activity and health tendencies, as well as some detraction against the physical-sportive trend. As to the teaching/learning processes, results show a particular evolution towards constructivist models. The findings are proof of the strengthening of some conceptions of physical education (including psycho-motor and basic motor skills and corporal expression) that are gaining momentum in the Ibero-American context, as well as the opening of the subject towards pedagogical models that grant greater importance for students in the teaching/learning process.

Keywords: physical education; didactics; learning; teaching; trends in physical education; pedagogical currents; pedagogical models

\section{Resumo}

Este artigo é resultado de uma pesquisa que visou elaborar um estado da arte sobre a produção de conhecimento cientifico em relação com o ensino/aprendizagem da educação física no contexto ibero-americano, entre os anos 2005 e 2016. A primeira parte apresenta a metodologia. Realizou-se uma busca da produção cientifica em diversas bases de dados por meio de frases compostas por palavras chave; aplicaram-se critérios de inclusão restringindo a busca só nas línguas espanhola, inglesa e portuguesa, e no período entre os anos 2005 e 2016. Os artigos científicos selecionados foram sometidos a uma matriz de análise categorial para o processo interpretativo. Na segunda parte, apresentam-se os resultados. Sobre as concepções da educação física, é evidente uma inclinação favorável para as tendências sociomotricidade e atividade física e saúde e algumas detrações frente à tendência físico-esportiva. Em relação com a produção dos processos de ensino/aprendizagem, assinala-se a evolução para os modelos construtivistas. As descobertas permitem evidenciar o fortalecimento de algumas concepções da educação física (incluindo a psicomotricidade, habilidades motrizes básicas e a expressão corporal) que são evidentes no contexto ibero-americano, assim como a apertura da disciplina para os modelos pedagógicos que oferecem maior participação dos estudantes no processo de ensino/aprendizagem.

Palavras chave: educação física; didática; aprendizagem; ensino; tendências na educação física; correntes pedagógicas; modelos pedagógicos

Fecha de recepción: 29 de julio de 2016

Fecha de aprobación: 3 de febrero de 2017

Para citar este artículo:

Ariza, J. y García, J. (2017). La enseñanza y el aprendizaje en Educación Física, un estado del arte de la producción científica en Iberoamérica entre 2005 y 2016. Lúdica Pedagógica, (25), 83-92. 


\section{INTRODUCCIÓN}

En la actualidad, abordar la didáctica de la educación física, convoca de manera sistemática a repensar todos los elementos que constituyen la propuesta de educar desde esta disciplina; para ello es necesario retomar los componentes del currículo, el cual, según De Zubiría (2000), "es la caracterización de los propósitos, los contenidos, la secuenciación, el método, los recursos didácticos y la evaluación. Cada uno de estos elementos resuelve una pregunta pedagógica diferente, pero interrelacionada con las demás" (p. 17).

La educación dispone de una variedad de vertientes que postulan una u otra forma como el mejor camino para concretar ese currículo y para desarrollar los procesos de enseñanza/aprendizaje; Not (1983) destaca cómo desde el siglo XVIII se han visto enfrentadas dos corrientes pedagógicas: la heteroestructurante y la autoestructurante, la primera enmarcada en el método tradicional; la segunda, se orienta hacia lo opuesto defendiendo la tesis de que el alumno mismo construye y determina su propia formación. En medio de estas dos posturas aparece la interestructuración, la cual reconoce a un estudiante activo en la producción de sus saberes, la importancia de su rol y lo trascendental del contexto (De Zubiría, 2006)

Estas corrientes pedagógicas están rodeadas y permeadas por teorías sociológicas, psicológicas (conductuales, cognitivas y constructivistas) y antropológicas que plantean un ideal de hombre y sociedad que la educación debe contribuir a formar (De Zubiría, 2000) y se plasman a través de modelos pedagógicos (Flórez, 2002).

La educación física no es ajena a esta multiplicidad de corrientes, teorías y modelos pedagógicos; el desarrollo de sus procesos de enseñanza/aprendizaje se ha visto influenciado por estas y estos desde los postulados de Mercurialis, Rousseau, Basedow, Pestalozzi, Muths, y de las escuelas gimnásticas alemana, sueca y francesa (Blázquez, 2001); hasta las tendencias actuales de la disciplina, como la físico-deportiva, la psicomotriz, la expresión corporal, la sociomotricidad, actividad física y salud, actividades en la naturaleza, y la tendencia alternativa (Zagalaz, 2001; Blázquez, 2001; Contreras 1998; Camacho, 2003); abriendo la posibilidad a un sinnúmero de opciones didácticas y a la aplicación de múltiples estilos de enseñanza (Mosston y Ashworth, 1993).
Ante este polifacético panorama pedagógico y curricular, la didáctica de la educación física, cuyo campo de estudio "está constituido por el conjunto de elementos que conforman el proceso de enseñanza/aprendizaje, así como los contextos en los que el mismo se da en referencia a las conductas motrices de relevancia educativa" (Contreras, 1998, p. 40) ha permanecido sujeto, al igual que la propia disciplina, al devenir y a los cambios impuestos por la cultura y las tendencias del momento; en este contexto de pluralidad de corrientes, teorías, modelos y apuestas pedagógicas que permean la educación física. El presente estudio estableció como objetivo elaborar un estado del arte sobre la producción de conocimiento científico en torno a la enseñanza/aprendizaje de la educación física en el contexto iberoamericano entre 2005 y 2016; para, de esta manera definir y caracterizar el contexto curricular actual de esta disciplina reconociendo sus propósitos, sus contenidos y estrategias de formación.

\section{METOdOLOgÍA}

El estudio se enmarcó en el paradigma histórico hermenéutico, se utilizó como estrategia el estado de arte y como técnica el análisis documental (Galeano, 2007); se realizó una búsqueda de artículos científicos y tesis doctorales en las bases de datos Redalyc, Scielo, Ebsco host, Proquest Education Journals y Dialnet; la citada búsqueda se centró en artículos científicos y tesis doctorales haciendo uso del descriptor booleano "AND", se utilizaron frases compuestas elaboradas de acuerdo con el tesauro de la Unesco: "Enseñanza AND Educación Física” y "Aprendizaje AND Educación Física"; la búsqueda se restringió a los idiomas español, portugués e inglés (Ensino AND educação física, aprendizagem AND educação física; Teaching AND physycal Education, Learning AND Physical education) y a la producción científica publicada entre 2005 y 2016. Para la selección final de los artículos se valoró el factor de impacto de las revistas en las que estos fueron publicados, utilizando el Scimago Journal Rank (SJR); los artículos correspondientes a revistas no indexadas allí fueron excluidos. Por último, se seleccionaron 62 artículos que se organizaron y codificaron a través de una ficha bibliográfica, la información contenida en los documentos se sistematizó en una matriz de Excel a partir de tres categorías establecidas con anterioridad: 
definición; educación física, enseña y/o aprendizaje, finalidad; proceso de enseñanza/aprendizaje de la educación física y la metodología.

\section{RESULTADOS}

\section{¿Qué es? Concepciones de la educación física (EF) y para qué. Finalidades y propósitos de sus procesos de enseñanza/aprendizaje}

Tendencia sociomotricidad

En el marco de esta tendencia, se reconoce en el uso de las prácticas corporales (capoeira) en las clases de EF una importante herramienta para rescatar manifestaciones culturales y reconocer el devenir cultural y político de su país (Brasil) (Da Costa, 2011); utilizando la EF para estudiar la cultura corporal (Rozengardt, 2005), sus aspectos históricos, sociológicos, antropológicos, pedagógicos (Romero, 2007; Cuevas, Pastor, González y Gil, 2010); psicológicos, convivenciales (Capllonch et al., citados por Capllonch y Figueras, 2012); la resolución de conflictos (Velásquez, Fraile y López, 2014; Velázquez et al., 2007; López, 2012; Sevil et al., 2015; Madrigal y Urrego, 2013; Gómez, 2005; Fernández et al., 2012; Poblete y Gamboa, 2013; López y Gea, 2010; Baena, Granero, Sánchez y Martínez, 2014 Molina y Antolin, 2008; Cárcamo, 2012); sin dejar de lado la inclusión de personas en condición de discapacidad (Robles, 2006; Fernández et al., 2012; Baena, Granero, Sánchez y Martínez, 2014 Molina y Antolín, 2008; Cárcamo, 2012).

\section{Tendencia actividad física (AF) y salud}

En cuanto a esta tendencia, se evidencia la importancia de la educación física como asignatura y disciplina que establece los lineamientos, contenidos y métodos para la AF (Reynaga, Landeros, Alonso y García, 2013), puesto que se le otorga el papel de proveedora (Gutiérrez, Ruiz y López, 2011), para la adopción de estilos de vida saludable en los jóvenes, por ser una etapa clave en la adopción y afianzamiento de hábitos. Por otro lado, se le asigna un rol fundamental al docente como facilitador o promotor de prácticas asertivas (Sevil et al., 2015; Moreno, Sicilia, Martínez y Alonso, 2008; Palao y Hernández, 2012; Baena y Granero 2015; Gutiérrez, Ruiz y López, 2011; Poblete y Gamboa, 2013; Ramírez, Mayorga y Cocca, 2014; Cuevas, García y Contreras, 2013; Monguillot, González, Guitert y Zurita, 2014; Castro, 2015; Pastor, 2007; Campos, Ries y Del Castillo, 2011).

\section{Tendencia físico-deportiva}

Según Valera, Ureña, Ruiz y Alarcón (2010) actualmente la integración del deporte como contenido curricular de la EF (Delgado, citado por Poblete y Gamboa, 2013) es muy marcada, tanto en la educación primaria, con una orientación lúdico-recreativa (Cechini, Fernández, González y Cechini, 2013; Velásquez, Fraile y López, 2014); como en la educación secundaria con más perfeccionamiento orientado hacia el desarrollo de capacidades físicas y habilidades específicas para cada deporte (Gómez et al., 2008; Lopes, 2014; Feu, Ibáñez y Gozalo, 2010). Los deportes que más se utilizan dentro de esta tendencia son los colectivos, preferiblemente los de invasión (Valera et al., 2010; Robles, Giménez y Abad, 2011; Otero, Carmona, Abornoz, Calvo y Díaz, 2014), encontrándose entre los más frecuentemente abordados, por lo menos en España, el balonmano, el baloncesto, el hockey, el fútbol y el fútbol sala.

La tendencia físico-deportiva no es muy bien aceptada por numerosos estudiosos, quienes reclaman no asignar a la EF funciones complementarias que no puede cumplir, siendo ilógico y estando demostrado que con dos horas semanales de EF no se puedan proporcionar los niveles de rendimiento que exige el deporte competitivo (Pastor, 2007). Así las cosas, López y Gea (2010) plantean que existen dos formas de entender y practicar la EF, una orientada al rendimiento y otra a la participación, situación que deriva en múltiples consecuencias a nivel curricular (contenidos, objetivos, metodologías, evaluación, etc.) que para el caso del rendimiento se inclina hacia un currículo por objetivos, con una metodología directiva que organiza el trabajo por grupos de élite motriz, y en cuya evaluación predomina la utilización de test de condición física y en pruebas de habilidades motrices y deportivas que dependen de ejecuciones técnicas determinadas.

Tendencia psicomotricidad y habilidades motrices básicas

La tendencia psicomotricidad en EF tiene sus raíces en los postulados de Le Boulch (Pastor, 2007) sobre el cuerpo, su imagen y percepción y que según Contreras (1998) se relaciona íntimamente con los bloques de contenidos del cuerpo, sus habilidades y destrezas.

Gómez, Jiménez y Sánchez (2015) resaltan en su estudio, cómo centrándose en la ejecución y organización de tareas motrices, la EF aporta al desarrollo de la autonomía y a la iniciativa personal; por su parte Madrigal y 
Urrego (2013) reconocen la relación entre el desarrollo motor y el desarrollo cognitivo afirmando que la EF contribuye desde la motricidad al desarrollo integral de los jóvenes (Velásquez, Fraile y López, 2014; Van der Kamp, Duivenvoorden, Kok y Van Hilvoorde, 2015; Cecchini, Fernández, Pallasa y Cecchini, 2012; Fernández, 2008; Gómez et al., 2008; López, 2008; Viciana, Mayorga y Cocca, 2013). Del mismo modo, Pastor (2007, citando a Le Boulch) resalta el movimiento como el principal medio de educación destacando las relaciones cuerpomovimiento acción y cuerpo-movimiento-conducta y fija (apoyado en Basquin) tres componentes dentro de la acción motriz: el esquema corporal, la representación psíquica del cuerpo y las experiencias afectivas del cuerpo; de este modo, en su estudio, Pastor (2007) anota que la EF no se puede abordar exclusivamente desde el plano motor; al intervenir es necesario tener en cuenta tanto la intención del proceso como la acción motriz para su desarrollo; en ese momento entran en juego el desarrollo psicológico, las funciones neuromotrices y las psíquicas, las capacidades mentales (análisis, síntesis, abstracción, simbolización, etc.) y lo corporal como medio para relacionarse con el mundo.

\section{Tendencia expresión corporal}

Poblete y Gamboa (2013) reconocen dentro de su estudio la teoría expresiva como una de las teorías implícitas en EF, en este contexto Gómez et al. (2008) plantean en su estudio la posibilidad de otorgarle un lugar prioritario a las situaciones expresivas de carácter comunicativo por medio del cuerpo y el movimiento del estudiante. Estas situaciones expresivas se constituyen en una opción didáctica para el desarrollo de los procesos de enseñanza/aprendizaje, considerando las necesidades particulares del género masculino y femenino (Sevil et al., 2015)

El panorama descrito evidencia la necesidad de que los docentes de EF sean competentes para el desarrollo de estas situaciones expresivas; al respecto, el estudio de Campos et al. (2011) precisa cómo trabajar la expresión corporal es la segunda competencia más valorada por los egresados maestros en EF de la Universidad de Sevilla.
¿Cómo?: se refiere particularmente a la manera como se producen o realizan los procesos de enseñanza/ aprendizaje de la Educación Física

Corriente heteroestructurante, pedagogía tradicional y conductismo en la educación física

En los documentos hallados se evidencia la corriente heteroestructurante y el modelo tradicional en las clases de EF; sobre todo en contextos donde la tendencia deportivista ocupa un lugar de preponderancia (López y Gea, 2010). Lo anterior se manifiesta en varios estudios donde se observa un débil estímulo por parte del docente en la capacidad creativa (Cárdenas, 2005, Gréhaigne y Godbout, 1997; Thorpe 1992, citados por Robles et al., 2011) y crítica del estudiante (Velásquez et al., 2007), además Robles et al. (2011) anotan que en el afán de conseguir la asimilación de gestos técnicos, la metodología tradicional es una constante, con actividades repetitivas y descontextualizadas (Valera et al., 2010; Calderón, Martínez y Hastie, 2013).

\section{Corriente autoestructurante y escuela}

activa en la educación física

Gómez et al. (2015) sostiene que las intervenciones docentes que promueven la autonomía (Baena y Granero, 2015) y la toma de decisiones obtienen mejores resultados en el desarrollo de habilidades motoras, práctica de actividad física, reducción de la desmotivación y del aburrimiento, aumento en los niveles de satisfacción, responsabilidad y aprendizaje adquirido (Hastie, Rudisill, y Wadsworth; Moreno, Parra y González-Cutre; Méndez, Cecchini, Fernández y González; Calderón, Martínez y Hastie; Gordon; Llopis et al.; Pascual et al.; Calderón, Martínez y Martínez; Lorente; citados por Gómez, Jiménez y Sánchez, 2015).

En ese mismo sentido trabajos como los de Gutiérrez et al. (2011), y Ruiz y Piéron (2013) ratifican la importancia de la orientación hacia metas de maestría (orientadas hacia la tarea) las cuales mejoran los niveles de motivación y autodeterminación puesto que los sujetos juzgan su nivel de capacidad a partir de un proceso de comparación con ellos mismos, mientras que cuando la meta es de competitividad, los individuos juzgan si son o no competentes, comparándose con los demás (Cervelló, Escartí y Balagué; Nicholls; Roberts y Treasure; citados por Ruiz y Piéron, 2013, p. 237). 
Corriente interestructurante y modelos

constructivistas en la educación física

Calderón, Martínez y Hastie (2013) afirman que los modelos de enseñanza aplicados en la EF han evolucionado de una tendencia magiocentrista a una que le da más valor al estudiante, que lo insta a la resolución de problemas, a la toma de decisiones y que promueve su autonomía. En ese contexto aparecen diferentes modelos entre los que los mismos autores destaca la educación deportiva y la enseñanza comprensiva de los deportes (Velázquez et al., 2007; Abad, Benito, Giménez y Robles, 2013; González, Gil, Contreras y Pastor, 2008; Alarcón et al., 2011; Robles, Giménez y Abad, 2011; Clemente, 2014; Castro, 2015; Vera, 2010), aprendizaje cooperativo (López, 2012; Velázquez, Fraile y López, 2014; Capllonch y Figueras, 2012; López, 2008), centrado en valores y crítico (López, 2012; López y Gea, 2010).

Estas corrientes interestructurantes y modelos constructivistas abren la posibilidad a la aplicación del juego en las clases de EF y de la mano de este a la creatividad, tal y como se evidencia en los estudios de Valero (2006), Rozengardt (2005) y Cechini et al. (2012); además promueven oportunidades de trabajo interdisciplinar, enfoques de aprendizaje integrado y propuestas de aprendizaje servicio (Del Valle, De la Vega y Rodríguez, 2015; Granero y Baena, 2015; Fernández, 2008; Coral y Lleixà, 2014; Martínez de Ojeda, Calderón y Campos, 2012; Corbatón et al., 2015); resaltándose también una presencia importante de metodologías fundamentadas en la orientación hacia la tarea y las metas de logro (Gómez, Jiménez y Sánchez, 2015; Cuevas et al., 2010; Navas, Soriano y Holgado, 2006; Cuevas, García y Contreras, 2013; Baena, Granero, Gómez y Abraldes, 2014; Moreno, Vera y Cervello, 2009). En esa misma línea, estudios como los de Baena et al. (2014); Palao y Hernández (2012); Sevil et al. (2015); Cea, Véliz, Aravena y Maureira (2014); Cechini et al. (2013); Vera, Moreno y Moreno (2009), y Gutiérrez y López (2011) evidencian los beneficios de teorías como la de la autodeterminación, las necesidades básicas y la cesión de responsabilidad.

\section{CONCLUSIONES}

Los hallazgos del presente estudio permiten evidenciar que tendencias de la EF como sociomotricidad, actividad física y salud toman fuerza exponiéndolas como las más adecuadas ante las limitaciones y desorientaciones de tendencias como la físico-deportiva y la de expresión corporal; la fuerte presencia de estas tendencias en la producción científica pone sobre la mesa concepciones que tienen más prioridad en el aspecto humano que en el técnico, que reconocen el cuerpo y el movimiento como actores indispensables en procesos de comunicación, interacción y transformación social.

Se muestra el modelo constructivista e interestructurante en EF como una posibilidad didáctica que está sobresaliendo y dejando atrás las corrientes heteroestructurantes, relegándolas prácticamente a una instrucción técnica fuera de contexto y realidad de juego en el marco de la tendencia deportivista. En detrimento de esas metodologías jerarquizadas y autoritarias, se observa el surgimiento de propuestas en torno a un rol del maestro como mediador y a favor del aprendizaje cooperativo y dialógico, del incremento de la motivación mediante la autodeterminación y las metas orientadas a la tarea y no al ego; una EF cuyos procesos de enseñanza/ aprendizaje parten de la cesión de responsabilidad, los valores, el pensamiento crítico, las habilidades interpersonales y el trabajo interdisciplinar, todo esto con influencia en lo cognitivo, lo social y lo afectivo.

\section{REFERENCIAS}

Abad, M., Benito, P., Giménez, F. y Robles, J. (2013). Fundamentos pedagógicos de la enseñanza comprensiva del deporte: Una revisión de la literatura. Cultura, Ciencia y Deporte, 8(23), 137-146.

Alarcón, L., Cárdenas, V., Piñar, M., Miranda, M. y Ureña, 0. (2011). La concepción constructivista como modelo explicativo del aprendizaje en los deportes de equipo. Universitas Psychologica, 10(2), 489-500.

Baena, A. y Granero, A. (2015). Educación física e intención de práctica física en tiempo libre. Revista Electrónica de Investigación Educativa, 17(3), 132-144.

Baena, A., Granero, A., Gómez, M. y Abraldes, J. (2014). Orientaciones de meta y clima motivacional según sexo y edad en educación física. Cultura, Ciencia y Deporte, 9(26), 119-128.

Baena, A., Granero, A., Sánchez, J. y Martínez, M. (2014). Modelo predictivo de la importancia y utilidad de la Educación Física. Cuadernos de Psicología del Deporte, 14(2), 121-130.

Blázquez, D. (2001). La educación física. Barcelona, España: INDE Publicaciones. 
Calderón, A., Martínez, D. y Hastie, P. (2013). Valoración de alumnado y profesorado de educación física tras la aplicación de dos modelos de enseñanza. RICYDE. Rev. Int. Cienc. Deporte., 32(9), 137-153.

Camacho, H. (2003). Pedagogía y didáctica de la educación física. Armenia, Colombia: Editorial Kinesis.

Campos, M., Ries, F. y Del Castillo, O. (2011). Análisis de las competencias adquiridas y utilizadas por los egresados maestros en Educación Física. International Journal of Sport Science, 7(24), 216-229.

Capllonch, M. y Figueras, S. (2012). Educación física y comunidades de aprendizaje. Estudios Pedagógicos, 38, 231-247.

Cárcamo, J. (2012). El profesor de educación física desde la perspectiva de los escolares. Estudios Pedagógicos, 38(1), 105-119.

Castro, S. (2015). Aprendizaje dialógico y educación física: Hacia una educación física y un deporte escolar promotores de salud, éxito escolar y cohesión social. Intangible Capital, 11(3), 393-417.

Cea, S., Véliz, C., Aravena, C. y Maureira, F. (2014). Percepción de los estudiantes de educación física de la UISEK de Chile hacia sus profesores. Cuadernos de Psicología del Deporte, 14(1), 23-28.

Cecchini, J., Fernández, J., González, C. y Cechini, C. (2013). Aplicaciones del modelo de autodeterminación en la educación física de primaria. Revista Latinoamericana de Psicologia, 45(1) 97-109.

Cecchini, J.A., Fernández, J.L., Pallasa, M. y Cecchini, C. (2012). El proceso de transferencia en el aprendizaje y desarrollo motor. Psicothema, 24(2), 205-210.

Clemente, M. (2014). Uma Visão Integrada do Modelo Teaching Games for Understanding: Adequando os Estilos de Ensino e Questionamento à Realidade da Educação Física. Revista Brasileira de Ciências do Esporte, 36(2) 587-601.

Contreras, J. (1998). Didáctica de la educación física. Barcelona, España: INDE Publicaciones.

Coral, J. y Lleixà, T. (2014). La enseñanza de la educación física mediante el enfoque educativo Clil: la resolución de los dilemas profesionales surgidos durante un proceso de investigación-acción. Movimento, 20(4), 1447-1472.

Corbatón, R., Moliner, M., Martí, M., Gil, J. y Chiva, 0. (2015). Efectos académicos, culturales, participativos y de identidad del aprendizaje-servicio en futuros maestros a través de la educación física. Profesorado, Revista de Currículum y Formación de Profesorado, 19(1), 280-297.
Cuevas, R., García, T. y Contreras, O. (2013). Perfiles motivacionales en educación física: una aproximación desde la teoría de las metas de logro $2 \times 2$. Anales de Psicología, 29(3), 685-69.

Cuevas, R., Pastor, J., González S. y Gil, P. (2010). Educación física intercultural, motivación y prejuicio racial: una aproximación desde la teoría de las metas de logro. Cuadernos de Psicología del Deporte, 10(Suple), 37-42.

Da Costa, P. (2011). Capoeira nas aulas de educação física: alguns apontamentos sobre processos de ensinoaprendizado de professores. Rev. Bras. Ciênc. Esporte, florianópolis, 33(4), 889-903.

De Zubiría, J. (2000). Los modelos pedagógicos. Tratado de pedagogía conceptual. Bogotá: Fundación Alberto Merani para el Desarrollo de la Inteligencia Cooperativa.

De Zubiría, J. (2006). Los modelos pedagógicos. Hacia una pedagogía dialogante. Bogotá: Cooperativa Editorial Magisterio.

Del Valle, S., De la Vega, R. y Rodríguez, M. (2015). Percepción de las competencias profesionales del docente de educación física en primaria y secundaria. Revista Internacional de Medicina y Ciencias de la Actividad Física del Deporte, 15(59), 507-526.

Fernández Abuín, J.P. (2008). El movimiento olímpico y las nuevas tecnologías en el área de Educación Física y Deporte a través del modelo didáctico de la webquest. Revista Internacional de Medicina y Ciencias de la Actividad Física y el Deporte, 8(29), 1-14.

Fernández, J.P. (2008). El movimiento olímpico y las nuevas tecnologías en el área de educación física y deporte a través del modelo didáctico de la webquest. Revista Internacional de Medicina y Ciencias de la Actividad Física y el Deporte, 8(29), 1-14.

Fernández, M., Sánchez, C., Jiménez, F., Navarro, V. y Anguera, M. (2012). Sistema de codificación y análisis de la calidad del dato para una intervención inclusiva en educación física. Revista de Psicología del Deporte, 21(1), 67-73.

Fernández, M., Sánchez, C.R., Jiménez, F., Navarro, V. y Anguera, M. (2012). Sistema de codificación y análisis de la calidad del dato para una intervención inclusiva en educación física. Revista de Psicología del Deporte, 21(1), 67-73.

Feu, S., Ibáñez, S. y Gozalo, M. (2010). La formación inicial de los entrenadores de balonmano para la enseñanza del deporte en la edad escolar. Cultura, Ciencia y Deporte, 5(14), 109-117.

Flórez, R. (2000). Hacia una pedagogía del conocimiento. Bogotá: McGraw-Hill. 
Flórez, R. (2002). Modelos pedagógicos y enseñabilidad de las ciencias. Actualidades Pedagógicas, 42, 17-26.

Galeano, M. (2007). Estrategias de investigación Social Cualitativa. El giro de la mirada. Medellín, Colombia: La Carreta Editorial.

Gómez, A. (2005). La enseñanza y el aprendizaje de los valores en la educación Deportiva. Revista Internacional de Medicina y Ciencias de la Actividad Física y el Deporte, 5(18), 89-99.

Gómez, A., Jiménez, F. y Sánchez, C. (2015). Desarrollo de la autonomía del alumnado de primaria en educación física a través de un proceso de investigación-acción. RICYDE. Revista Internacional de Ciencias del Deporte, 11(42), 310-328.

Gómez, A., Díez, L.J., Fernández, J.M., Gorrín, A., Pacheco, J.J. y Sosa A., G. (2008). Nueva propuesta curricular para el área de Educación Física en la Educación Primaria. Revista Internacional de Medicina y Ciencias de la Actividad Física y el Deporte, 8(29), 93-108.

González, S., Gil, P., Contreras, 0. y Pastor, J. (2008). Propuesta de formación permanente del profesorado de educación física sobre deportes de invasión. Perfiles educativos, 30(121), 7-124.

Granero, A. y Baena, A. (2015). Diseños de aprendizaje basados en las TIC (Moodle 2.0 y Mahara) para contenidos de Anatomía, Fisiología y Salud en las clases de Educación Física escolar. International Journal of Morphology, 33(1), 375-381.

Gutiérrez, M. y López, E. (2011). Percepción de las estrategias que emplean los profesores para mantener la disciplina, razones de los alumnos para ser disciplinados y comportamiento en educación física. International Journal of Sport Science, 7(22), 24-38.

Gutiérrez, M., Ruiz, L. y López, E. (2011). Clima motivacional en educación física: concordancia entre las percepciones de los alumnos y las de sus profesores. Revista de Psicología del Deporte Universität de les liles Balears, 20(2), 321-335.

Lopes, E. (2014). Quando "rola a bola": reflexões sobre as práticas futebolísticas e a forma escolar nas aulas de educação física. Rev. Bras. Ciênc. Esporte, florianópolis, 36(2), 501-513.

López, V.M. (2012). Didáctica de la educación física, desigualdad y transformación social. Estudios Pedagógi$\cos , 38(1), 155-176$.

López, V.M. (enero-abril de 2008). La importancia del trabajo colaborativo para el tratamiento de la motricidad infantil. Una propuesta integral a partir del aprendizaje inducido. Revista Española de Pedagogía, $239,137-150$.
López, V.M. y Gea, J.M. (2010). Innovación, discurso y racionalidad en Educación Física. Revisión y prospectiva. Revista Internacional de Medicina y Ciencias de la Actividad Física y el Deporte, 10(38), 245-270.

Madrigal, A. y Urrego, A. (2013). La educación física renovada desde la investigación educativa. Revista Lasallista de Investigación, 10(2), 69-76.

Martínez de Ojeda, D., Calderón, A. y Campos, A. (2012). Percepción de aprendizaje y satisfacción en una unidad didáctica integrada mediante el modelo de educación deportiva. Cultura, ciencia y deporte, 7(21) 163-172.

Molina, J. y Antolín, l. (2008). Las competencias básicas en educación física: una valoración crítica. Cultura, Ciencia y Deporte, 3(8), 81-86.

Monguillot, M., González, C., Guitert, M. y Zurita, C. (2014). Mobile learning: a collaborative experience using QR codes. Mobile Learning Applications in Higher Education [Special Section]. Revista de Universidad y Sociedad del Conocimiento, 11(1) 175-191.

Moreno, J., Sicilia, A., Martínez, C. y Alonso, N. (2008). Coeducación y climas de aprendizaje en educación física. Aportaciones desde la teoría de metas de logro. Revista Internacional de Ciencias del Deporte, 11(4), 42-64.

Moreno, J.A., Vera, J.A. y Cervello, E. (2009). Efectos de la cesión de responsabilidad de la evaluación en la motivación y la competencia percibida en el aula de educación física. Revista de Educación, 348, 423-440.

Mosston, M. y Ashworth, S. (1993). La enseñanza de la educación física. la reforma de los estilos de enseñanza. Barcelona, España: Editorial Hispano-Europea.

Navas, L., Soriano, J.A. y Holgado, F.P. (2006). Orientaciones de meta en las clases de educación física: un análisis centrado en la educación secundaria obligatoria. Revista de Psicología del Deporte, 15(2), 167-181.

Not, L. (1983). Las pedagogías del conocimiento. México D.F.: Fondo de Cultura Económica.

Ortiz, A. (2011). Hacia una nueva clasificación de los modelos pedagógicos: el pensamiento configuracional como paradigma científico y educativo del siglo XXI. Revista Praxis, 7, 121-137.

Otero, F.M., Carmona, J., Albornoz, M., Calvo, A. y Díaz, J.A. (2014). Metodología de enseñanza de los deportes de invasión en primaria. Revista Internacional de Medicina y Ciencias de la Actividad Física y el Deporte, 14(53), 69-87.

Palao, J. y Hernández, E. (2012). Validación de un instrumento para valorar la percepción del aprendizaje y el nivel de diversión del alumno en educación física. El semáforo. Cuadernos de Psicología del Deporte, 12(1), 25-32. 
Pastor, J.L. (2007). Fundamentación epistemológica e identidad de la educación física. Profesorado. Revista de currículum y formación del profesorado, 11(2),1-17.

Poblete, C. y Gamboa, R. (2013). Teorías implícitas y proyectos educativos: una visión paradigmática de la educación física. Educere, 17(58), 465-474.

Ramírez, J., Mayorga, D. y Cocca, A. (2014). Modelo de aprendizaje exitoso en educación física y su mantenimiento. Estudio del efecto del refuerzo intermitente sobre la condición física Facultad de Ciencias de la Actividad Física y el Deporte. Revista Iberoamericana de Psicología del Ejercicio y el Deporte, 9(1),155-171.

Reynaga, P., Landeros, A., Alonso, T. y García, A. (2013). Presencia curricular de la psicología de la actividad física y del deporte en los planes de estudio de pregrado y posgrado de educación física versus psicología, en México. Revista Brasileira de Ciências do Esporte, 35(4) 847-863.

Robles, J. (2006). Judo para ciegos como contenido novedoso en las clases de educación física. Unidad Didáctica. Revista Internacional de Medicina y Ciencias de la Actividad Física y el Deporte, 6(22), 75-86.

Robles, J., Giménez, F.J. y Abad, M.T. (2011). Metodología utilizada en la enseñanza de los contenidos deportivos durante la E.S.O. Revista Internacional de Medicina y Ciencias de la Actividad Física y el Deporte, 11(41), 35-57.

Romero, C. (2007). Delimitación del campo didáctico de la educación física y de su actividad científica. Profesorado. Revista de Currículum y Formación del Profesorado, 11(2), 1-45.

Rozengardt, R. (2005). Aprendizaje, juego y niño: un modo de entender la educación física. Revista Brasileira de Ciências do Esporte, 26(3) 111-126.

Ruiz, J.F. y Piéron, M. (2013). Orientaciones de meta en Educación Física y nivel de actividad físico-deportiva en estudiantes mexicanos. Universitas Psychologica, 12(1), 235-247.

Sevil, J., Abós, A., Julian, J.A., Murillo, B. y García-González, L. (2015). Género y motivación situacional en educación física: claves para el desarrollo de estrategias de intervención. RICYDE. Revista Internacional de Ciencias del Deporte, 41(11), 281-296.
Valera, S., Ureña, N., Ruiz, E. y Alarcón, F. (2010). La enseñanza de los deportes colectivos en Educación Física en la E.S.O. Revista Internacional de Medicina y Ciencias de la Actividad Física y el Deporte, 10(40), 502-520.

Valero, A. (2006). La evaluación en el modelo de enseñanza ludotécnico para la iniciación a la práctica del atletismo dentro del contexto escolar. Cultura, ciencia y deporte, 2(5) 89-94.

Van der Kamp, J., Duivenvoorden, J., Kok, M. y Van Hilvoorde, I. (2015). Aprendizaje de habilidades/ destrezas motoras en grupos: propuestas para la aplicación de aprendizaje implícito, autocontrolado y retroalimentado. RICYDE. Revista Internacional de Ciencias del Deporte, 39(11), 33-47.

Velázquez, C., Fraile, A. y López, V. (2014). Aprendizaje cooperativo en educación física. Movimento, 20(1), 239-259.

Velázquez, R., Hernández, J., Garoz, I., López, C., López, M., Maldonado, A., Martínez, M., Villagra, A. y Castejón, J. (2007). Calidad de enseñanza en educación física y deportiva y discurso docente: el caso de la comunidad de Madrid. Revista de Educación, 344, 447-467.

Vera, J. (2010). Autobiografía, identidad docente y conocimiento didáctico en la enseñanza de la educación física. Revista Complutense de Educación, 21(2), 423-44.

Vera, J.A., Moreno, R. y Moreno, J. (2009). Relaciones entre la cesión de responsabilidad en la evaluación y la percepción de igualdad en la enseñanza de la educación física escolar. Cultura, Ciencia y Deporte, 4(10), 25-31.

Viciana, J., Mayorga, D. y Cocca, A. (2013). Modelo de aprendizaje exitoso en educación física y su mantenimiento. Estudio del efecto del refuerzo intermitente sobre la condición física. Revista Iberoamericana de Psicología del Ejercicio y el Deporte, 9(1), 155-171.

Zagalaz, L. (2001). Corrientes y tendencias de la educación física. Barcelona, España: INDE Publicaciones. 